\title{
Preventing Pollution Attacks in Multi-source Network Coding
}

\author{
Shweta Agrawal ${ }^{1, \star}$, Dan Boneh ${ }^{2, \star \star}$, \\ Xavier Boyen ${ }^{3, \star \star \star}$, and David Mandell Freeman ${ }^{2, \dagger}$ \\ 1 University of Texas at Austin, USA \\ shweta.a@gmail.com \\ 2 Stanford University, USA \\ \{dabo, dfreeman\}@cs.stanford.edu \\ 3 Université de Liège, Belgium \\ xb@boyen.org
}

\begin{abstract}
Network coding is a method for achieving channel capacity in networks. The key idea is to allow network routers to linearly mix packets as they traverse the network so that recipients receive linear combinations of packets. Network coded systems are vulnerable to pollution attacks where a single malicious node floods the network with bad packets and prevents the receiver from decoding correctly. Cryptographic defenses to these problems are based on homomorphic signatures and MACs. These proposals, however, cannot handle mixing of packets from multiple sources, which is needed to achieve the full benefits of network coding. In this paper we address integrity of multi-source mixing. We propose a security model for this setting and provide a generic construction.
\end{abstract}

\section{Introduction}

Network coding 316 is an elegant technique that replaces the traditional "store and forward" paradigm of network routing by a method that allows routers to transform the received data before re-transmission. It has been established that for certain classes of networks, random linear coding is sufficient to improve throughput [1]. In addition, linear network codes offer robustness and adaptability and have many practical applications (in wireless and sensor networks, for example) [10. Due to these advantages, network coding has become very popular.

On the other hand, networks using network coding are exposed to problems that traditional networks do not face. A particularly important instance of this is the pollution problem: if some routers in the network are malicious and forward invalid combinations of received packets, then these invalid packets get

* Supported by DARPA IAMANET.

** Supported by DARPA IAMANET and NSF.

$\star \star \star$ Work done in part at Stanford, with support from DARPA IAMANET and NSF.

${ }^{\dagger}$ Work done in part at CWI and Univ. Leiden. Supported by an NSF International Research Fellowship and the NSF Office of Multidisciplinary Activities. 
mixed with valid packets downstream and quickly pollute the whole network. In addition, the receiver who obtains multiple packets has no way of ascertaining which of these are valid and should be used for decoding. Indeed, using even one invalid packet during the decoding process causes all the messages to be decoded wrongly. For a detailed discussion of pollution attacks, we refer the reader to 41912 .

To prevent the network from being flooded with invalid packets, it is desirable to have "hop-by-hop containment." This means that even if a bad packet gets injected into the network, it is detected and discarded at the very next hop. Thus, it can be dropped before it is combined with any other packets, preventing its pollution from spreading.

Hop-by-hop containment cannot be achieved by standard signatures or MACs. As pointed out in [1], signing the message packets does not help since recipients do not have the original message packets and therefore cannot verify the signature. Nor does signing the entire message prior to transmission work, because it forces the recipient to decode exponentially many subsets of received packets to find a decoded message with a consistent signature. Thus, new integrity mechanisms are needed to mitigate pollution attacks.

Previous Work. Security of network coding has been considered from both the information-theoretic and cryptographic perspectives. In the former, the adversary is modelled as having control over a limited number of links in the network. Such approaches, though useful for wireline networks, have limited application in wireless networks. For a detailed discussion of these techniques, see e.g. 691314. Cryptographic techniques have also been proposed, e.g. in 7,17,194. These authors construct digital signatures for signing a linear subspace. If $V$ is a subspace and $\sigma$ its signature, then there is a verification algorithm which accepts the pair $(\mathbf{v}, \sigma)$ for all $\mathbf{v} \in V$, but it is difficult to construct a vector $\mathbf{y} \notin V$ for which the pair $(\mathbf{y}, \sigma)$ verifies. An alternative approach is to use a MAC (instead of a signature) for integrity of a linear subspace; see [118.

While the signature and MAC schemes in [7/7/1941] are elegant, they are quite limited: they only allow routers to combine vectors from a single sender. (Furthermore, the constructions of 7/17/9 require a new public key to be generated for each file, thus hurting efficiency.) Traditional network coding assumes a network where many senders simultaneously send messages and network routers linearly combine vectors from multiple senders. This setting is essential in showing that network coding can improve the efficiency of 802.11 wireless networks 15 .

Our Contribution. Our goal is to construct a signature mechanism that provides integrity when network routers combine packets from many sources. This problem is considerably harder than the single source problem. First, defining security is more difficult. It is necessary to model "insider" attacks where the attacker controls network routers as well as some senders. The attacker's goal is to generate valid signatures on mixed packets; after decoding these packets the recipient believes that an honest sender sent a message $M^{*}$ that was never sent by the honest sender. 
More precisely, if there are $s$ senders in the network, we allow the attacker to control $s-1$ of them. Furthermore, the attacker can mount a chosen message attack on the single honest sender. The attacker's goal is to generate a mixed packet with a valid signature that after decoding corresponds to an existential forgery on the single honest sender.

In Section 3 we show that a natural generalization of the single-sender security model in [4] to the multi-sender setting results in a model that cannot be satisfied. We do this by constructing a generic attack against an abstract multi-source network coding signature scheme. In Section 4 we present a security model that captures the constraints of the multi-sender problem. Our model retains the desirable properties of the single-source model, such as hop-by-hop containment of forged packets, and is achievable.

In Section 5 we present a construction satisfying our security model. We give a generic construction from a new primitive called a vector hash, which captures the properties of homomorphic hashing that are necessary to produce secure signatures. In the full version of this paper 2] we show how to instantiate the construction based on the discrete logarithm assumption. We also prove a lower bound that shows that our model necessitates a relatively space-inefficient construction; our discrete log scheme (asymptotically) achieves this lower bound.

\section{Network Coding}

We refer the reader to [16] for a detailed introduction to network coding. Here we present a brief overview for completeness; this description describes the operation of a network coding system and is independent of any security model. We model a network as a directed graph consisting of a set of vertices (or nodes) $V$ and a set of edges $E$. We assume the graph is connected. A node that only transmits data is called a source node. We start with the basic model, in which one source wishes to transmit one file $F$ through the network. The source interprets the data in $F$ as a set of $m$ vectors $\hat{\mathbf{v}}_{1}, \ldots, \hat{\mathbf{v}}_{m}$ in an $n$-dimensional vector space over a finite field $\mathbb{F}_{p}$. (The prime $p$ and the dimensions $n$ and $m$ are fixed parameters in the system.) We sometimes refer to individual vectors as blocks or packets. The source then appends a unit vector of length $m$ to the vectors $\hat{\mathbf{v}}_{i}$ to create $m$ augmented vectors $\mathbf{v}_{1}, \ldots, \mathbf{v}_{m}$ given by

$$
\mathbf{v}_{i}=(-\hat{\mathbf{v}}_{i}-\overbrace{\underbrace{0, \ldots, 0,1}_{i}, 0, \ldots, 0}^{m}) \in \mathbb{F}_{p}^{n+m} .
$$

The augmented vectors comprise the data to be transmitted through the network. We call the first $n$ entries of the vector $\mathbf{v}_{i}$ the data component and the last $m$ entries the augmentation component.

The "coding" part of network coding works as follows: an intermediate node in the network receives some set of vectors $\mathbf{w}_{1}, \ldots, \mathbf{w}_{\ell}$, chooses $\ell$ random elements $\beta_{i} \in \mathbb{F}_{p}$, and transmits the vector $\mathbf{y}=\sum_{i=1}^{\ell} \beta_{i} \mathbf{w}_{i}$ along its outgoing edges. The key property of the augmentation is that the augmentation component contains exactly the linear combination coefficients used to construct $\mathbf{y}$. That is, we know 
that $\mathbf{y}=\sum_{i=1}^{m} y_{n+i} \mathbf{v}_{i}$ even though the intermediate node may never see the $\mathbf{v}_{i}$. This property allows any node that receives a set of $m$ linearly independent vectors $\mathbf{y}_{1}, \ldots, \mathbf{y}_{m}$ to recover the original $\mathbf{v}_{i}$. Specifically, if we let $D$ be $m \times n$ matrix whose $i$ th row consists of the data component of $\mathbf{y}_{i}$, and $A$ be the $m \times m$ matrix whose $i$ th row consists of the augmentation component of $\mathbf{y}_{i}$, then the rows of $A^{-1} D$ are exactly the initial vectors $\hat{\mathbf{v}}_{i}$.

Since network coding consists of linearly combining vectors, the subspace spanned by the (augmented) vectors of a file remains invariant under network operations. Hence we can equivalently consider a file to be represented by the subspace spanned by the vectors that comprise it.

Notation. We use $n$ to denote the dimension of the data space and $m$ to denote the dimension of a vector subspace that represents a single file. The number of files in the system is denoted by $f$. For $\mathbf{v} \in \mathbb{F}_{p}^{n+\ell}$, we will use $\hat{\mathbf{v}}$ to denote the data component of $\mathbf{v}$, i.e., the first $n$ coordinates of $\mathbf{v}$, and $\bar{\beta}_{\mathbf{v}}$ to denote the augmentation component of $\mathbf{v}$, i.e., the remaining $\ell$ coordinates. When we use a vector space $V$ as input to or output of an algorithm we assume that $V$ is described by an explicit basis $\left\{\mathbf{v}_{1}, \ldots, \mathbf{v}_{\ell}\right\}$. Such a basis is properly augmented if for $i=1, \ldots, \ell$, the augmentation component $\bar{\beta}_{\mathbf{v}_{i}}$ is the unit vector $\mathbf{e}_{i}$ with a 1 in the $i$ th place.

We will refer to the augmented vectors that the source wishes to transmit as primitive vectors. Here, "primitive" alludes to the fact that these vectors have not been mixed with any other; their augmentation components are unit vectors. Aggregate vectors, on the other hand, refer to vectors that have been formed as a result of linearly combining primitive or other aggregate vectors.

\subsection{Multiple Sources, Multiple Files}

In general networks may have multiple sources, each of which can transmit multiple files into the network. We now describe this situation, assuming that all nodes in the network are honest. In principle the network coding setup is the same as in the single-source situation described above, but there is some more bookkeeping to do. This bookkeeping is implicit in previous work that considers multiple sources (e.g. [16]); here we give an explicit description that we will use in our discussion of security. The complication arises from the fact that the intermediate nodes wish to combine vectors from files produced by different sources, but each source knows nothing of what the other sources are doing.

In the single source case, each file is associated with a file identifier id. The identifier allows the receiver to group together packets that belong to the same file. This prevents, for example, delayed honest packets from a previous file transmission from being decoded along with the current file's vectors. Hence each vector (primitive or aggregate) that traverses the system carries with it the identifier of the file it belongs to.

In the multi-source case, the file identifier id plays an even more crucial role - it allows the intermediate nodes to combine vectors arising from different files. In this scenario, an aggregate vector may be associated with multiple files, 
and the identifier attached to an aggregate vector $\mathbf{v}$ must carry with it the identifiers of all of the files whose vectors went into making $\mathbf{v}$. Upon receiving two vectors, where each vector contains a (probably different) list of identifiers $\overline{\mathrm{id}}$, an intermediate node will need to "merge" the lists of identifiers to a common list and adjust the two vectors' augmentation components so that they can be linearly combined.

For example, suppose a node receives two vectors $\mathbf{v}_{1}, \mathbf{v}_{2} \in \mathbb{F}_{p}^{n+m}$ with identifiers $\mathrm{id}_{1}$ and $\mathrm{id}_{2}$, respectively. Splitting $\mathbf{v}_{i}$ into its data and augmentation components, we write $\mathbf{v}_{i}=\left(\hat{\mathbf{v}}_{i}, \mathbf{a}_{i}\right)$. If $\mathrm{id}_{1}=\mathrm{id}_{2}$ then the vectors come from the same file and the situation is analogous to the single source case and no additional adjustment is needed. However, if $\mathrm{id}_{1} \neq \mathrm{id}_{2}$ then the vectors came from different files and we must introduce additional augmentation before we can linearly combine the vectors. In this case we define $\mathbf{v}_{1}^{\prime}=\left(\hat{\mathbf{v}}_{1}, \mathbf{a}_{1}, \mathbf{0}\right)$ and $\mathbf{v}_{2}^{\prime}=\left(\hat{\mathbf{v}}_{2}, \mathbf{0}, \mathbf{a}_{2}\right) \in \mathbb{F}_{p}^{n+2 m}$, where $\mathbf{0}$ denotes a length- $m$ zero vector. Thus when we compute a linear combination $\mathbf{v}=a \mathbf{v}_{1}^{\prime}+b \mathbf{v}_{2}^{\prime}$, the data components are mixed together but the augmentation coefficients remain separate. We can then use the identifier $\overline{\mathrm{id}}=\left(\mathrm{id}_{1}, \mathrm{id}_{2}\right)$ to indicate which set of augmentation coefficients correspond to which file.

More generally, we define an algorithm Merge that merges the lists of identifiers contained in aggregate vectors and adjusts the vectors' augmentations. This algorithm is intrinsic to the multiple-source setting: the algorithm does not itself linearly combine vectors, but rather it prepares aggregate vectors (coming from different sources, made up of different files) to be mixed together. If $\mathbf{v} \in \mathbb{F}_{p}^{n+m f}$ is an aggregate vector, we continue to call the first $n$ entries of $\mathbf{v}$ the data component; we call the rest of $\mathbf{v}$ the augmentation component, and we divide the augmentation component into $f$ augmentation blocks of length $m$. (Here and in the remainder of the paper we assume for simplicity the dimension $m$ is the same for each file and is publicly known; the generalization to variable dimension is straightforward.)

\section{Algorithm 1. (Merge)}

Input: lists of identifiers $\overline{\mathrm{id}}_{1}, \overline{\mathrm{id}}_{2}$ of lengths $f_{1}, f_{2}$, respectively, with no repeated entries, and vectors $\mathbf{w}_{i} \in \mathbb{F}_{p}^{n+m f_{i}}$ for $i=1,2$.

Output: vectors $\mathbf{w}_{1}^{\prime}, \mathbf{w}_{2}^{\prime} \in \mathbb{F}_{p}^{n+m f^{\prime}}$ and a list of identifiers $\overline{\mathrm{id}}^{\prime}$ of length $f^{\prime}$.

1. Let $\overline{\mathrm{id}}^{\prime}$ be the list whose entries are the union of the elements of $\overline{\mathrm{id}}_{1}$ and $\overline{\mathrm{id}}_{2}$, ordered in some pre-determined way (e.g. lexicographically). Let $f^{\prime}$ be the length of $\overline{\mathrm{id}}^{\prime}$.

2. For $i$ in 1,2 , define $\mathbf{w}_{i}^{\prime} \in \mathbb{F}_{p}^{n+m f^{\prime}}$ by setting the data component of $\mathbf{w}_{i}^{\prime}$ equal to the data component of $\mathbf{w}_{i}$, and for $j$ in $1, \ldots, f^{\prime}$, setting the $j$ th augmentation block of $\mathbf{w}_{i}^{\prime}$ as follows:

- If the $j$ th element of $\overline{\mathrm{id}}^{\prime}$ is the $k$ th element of $\overline{\mathrm{id}}_{i}$, the $j$ th augmentation block of $\mathbf{w}_{i}^{\prime}$ is equal to the $k$ th augmentation block of $\mathbf{w}_{i}$.

- If the $j$ th element of $\overline{\mathrm{id}}^{\prime}$ is not an element of $\overline{\mathrm{id}}_{i}$, the $j$ th augmentation block of $\mathbf{w}_{i}^{\prime}$ is $\mathbf{0}$.

3. Output the list $\overline{\mathrm{id}}^{\prime}$ and the vectors $\mathbf{w}_{1}^{\prime}, \mathbf{w}_{2}^{\prime}$. 
The intermediate node can now compute a random linear combination $\mathbf{y}$ of the $\mathbf{w}_{1}^{\prime}$ and $\mathbf{w}_{2}^{\prime}$ and use the list $\overline{\mathrm{id}}^{\prime}$ as the identifier component of the signature on y. (In the example above we executed this algorithm on two vectors each with an identifier list of length $f_{i}=1$.)

We also define an algorithm called MergeSpaces that uses the Merge algorithm to combine two files described as vector spaces.

\section{Algorithm 2. (MergeSpaces)}

Input: disjoint lists of identifiers $\overline{\mathrm{id}}_{1}, \overline{\mathrm{id}}_{2}$ and two vector spaces $V=$ $\operatorname{span}\left(\mathbf{v}_{1}, \ldots, \mathbf{v}_{k}\right) \subset \mathbb{F}_{p}^{n+k}$ and $W=\operatorname{span}\left(\mathbf{w}_{1}, \ldots, \mathbf{w}_{\ell}\right) \subset \mathbb{F}_{p}^{n+\ell}$.

Output: a subspace $Z \subset \mathbb{F}_{p}^{n+k+\ell}$ and an identifier $\overline{\mathrm{id}}^{\prime}$.

1. Let $B$ be the set of nonzero vectors produced by

$$
\begin{gathered}
\operatorname{Merge}\left(\overline{\mathrm{id}}_{1}, \overline{\mathrm{id}}_{2}, \mathbf{v}_{1}, \mathbf{0}\right), \ldots, \operatorname{Merge}\left(\overline{\mathrm{id}}_{1}, \overline{\mathrm{id}}_{2}, \mathbf{v}_{k}, \mathbf{0}\right), \\
\operatorname{Merge}\left(\overline{\mathrm{id}}_{1}, \overline{\mathrm{id}}_{2}, \mathbf{0}, \mathbf{w}_{1}\right), \ldots, \operatorname{Merge}\left(\overline{\mathrm{id}}_{1}, \overline{\mathrm{id}}_{2}, \mathbf{0}, \mathbf{w}_{\ell}\right) .
\end{gathered}
$$

2. Let $\overline{\mathrm{id}}^{\prime}$ be the identifier output by any of the calls to Merge in Step (1).

3. Output $Z=\operatorname{span}(B)$ and $\overline{\mathrm{id}}^{\prime}$.

By applying MergeSpaces repeatedly using concatenated lists of identifiers, the algorithm generalizes to take any number of vector spaces and identifiers as input. The decoding operation works as before: given a set of vectors whose (merged) augmentation components form a full-rank matrix, we can recover the original data vectors by inverting this matrix.

\section{Signatures and File Identifiers}

For single sources, a network coding signature scheme consists of three algorithms, Setup, Sign, and Verify, whose functionality correspond to the usual notions for a signature scheme. In this setting, the Sign algorithm produces signatures on a vector space, and the Verify algorithm checks whether the signature is valid on a given vector. In addition, both Sign and Verify take as additional input a file identifier id, which binds a signature to a file. Informally, the correctness condition is that if $\sigma$ is a signature on a vector space $V$ with identifier id, then for all $\mathbf{v} \in V$, Verify(id, v, $\sigma$ ) outputs "accept." (For formal definitions, see [4, Section 3.1].)

For multiple sources, we need to add an additional algorithm Combine that will be used by intermediate routers to produce signatures on vectors that are linear combinations of vectors from different files. More precisely, Combine takes as input two tuples $\left(\mathbf{v}_{i}, \overline{\mathrm{id}}_{i}, \sigma_{i}, a_{i}\right)$ for $i=1,2$, where $\mathbf{v}_{i}$ are vectors, $\overline{\mathrm{id}}_{i}$ are (lists of) identifiers, $\sigma_{i}$ are signatures, and $a_{i}$ are network coding coefficients. The algorithm outputs a signature $\sigma^{\prime}$. The correctness condition is that if $\sigma_{i}$ is a valid signature on $\mathbf{v}_{i}$ with identifier $\overline{\mathrm{id}}_{i}$ for $i=1,2$, then $\sigma^{\prime}$ is a valid signature on $a_{1} \mathbf{v}_{1}^{\prime}+a_{2} \mathbf{v}_{2}^{\prime}$ with identifier $\overline{\mathrm{id}}^{\prime}$, where $\mathbf{v}_{1}^{\prime}, \mathbf{v}_{2}^{\prime}, \overline{\mathrm{id}}^{\prime}$ are output by Merge $\left(\overline{\mathrm{id}}_{1}, \overline{\mathrm{id}}_{2}, \mathbf{v}_{1}, \mathbf{v}_{2}\right)$. 
In the single-source setting, the Sign algorithm takes id as input. Thus, a vector $\mathbf{v}$ carries a pair (id, $\sigma$ ) where id is the file identifier chosen arbitrarily, and $\sigma$ is generated by Sign. In the multi-source case however, allowing senders to pick file identifiers gives them the ability to frame other users in the system, so that receiver Bob can be made to believe that user Alice sent him a packet which, in fact, Alice did not. In most network coded systems with multiple senders, such as BitTorrent 8, insider attacks form the real threat, so this attack has significant practical implications. Fortunately, this attack can be thwarted by enforcing that the file identifiers be cryptographically verifiable. In the subsequent sections, we will formalize these notions. We first describe the attack, and then use the intuition gained from the attack to construct a framework that can circumvent it.

\subsection{Generic Attack (For Arbitrary File Identifiers)}

Here we construct an attack against an abstract multi-source network coding signature scheme that consists of the algorithms Setup, Sign, Combine, Verify discussed above. We make no assumptions about these algorithms beyond their functionality. We show that it is impossible to achieve hop-by-hop containment if the identifier id is chosen arbitrarily by the sender and is given as input to the Sign algorithm. We construct a generic attack in which an intermediate node is fooled into accepting invalid packets as valid. As mentioned before, the attack is an "insider" attack where one of the senders is malicious. The malicious sender can assign two different vector spaces the same id and sign both using his secret key. An intermediate node has no hope of ever detecting this, since two packets constructed using these two vector spaces are both individually valid, but they are not pairwise valid, and can cause the receiver to incorrectly decode an honest user's message. We make this formal below.

We explain the attack with subspace dimension $m=1$; the attack easily generalizes to arbitrary $m$. In our system, the honest sender is Alice, the receiver is Bob, and the malicious user is Mallet.

Honest User Alice. Alice wishes to send a file described as a single nonzero vector $\hat{\mathbf{v}}_{1} \in \mathbb{F}_{p}^{n}$. She sets $\mathbf{v}_{1}=\left(\hat{\mathbf{v}}_{1}, 1\right)$, chooses a file identifier $\mathrm{id}_{\alpha}$ and uses her secret key $\mathbf{s k}_{\alpha}$ to create a signature $\tau_{1}$ on the one-dimensional subspace $V_{1} \subset \mathbb{F}_{p}^{n+1}$ spanned by $\mathbf{v}_{1}$, with identifier $\mathrm{id}_{\alpha}$. Then she transmits the packet $P_{1}=\left(\mathbf{v}_{1}, \mathrm{id}_{\alpha}, \tau_{1}\right)$.

Malicious User Mallet. Mallet receives $P_{1}$ and does the following:

1. Generate a key pair $\left(\mathrm{sk}_{\mu}, \mathrm{pk}_{\mu}\right)$.

2. Pick two vectors $\hat{\mathbf{v}}_{2}, \hat{\mathbf{v}}_{3} \in \mathbb{F}_{p}^{n}$ such that the set $\left\{\hat{\mathbf{v}}_{1}, \hat{\mathbf{v}}_{2}, \hat{\mathbf{v}}_{3}\right\}$ are linearly independent. Let $V_{2}, V_{3}$ be the subspaces of $\mathbb{F}_{p}^{n+1}$ spanned by $\mathbf{v}_{2}=\left(\hat{\mathbf{v}}_{2}, 1\right)$ and $\mathbf{v}_{3}=\left(\hat{\mathbf{v}}_{3}, 1\right)$, respectively.

3. Choose an identifier $\mathrm{id}_{\mu} \neq \mathrm{id}_{\alpha}$, and use the key sk $\mathrm{s}_{\mu}$ to compute signatures $\tau_{2}, \tau_{3}$ on subspaces $V_{2}, V_{3}$ with identifier id ${ }_{\mu}$. Create the packets $P_{2}=$ $\left(\mathbf{v}_{2}, \mathrm{id}_{\mu}, \tau_{2}\right), P_{3}=\left(\mathbf{v}_{3}, \mathrm{id}_{\mu}, \tau_{3}\right)$.

4. Run Merge on $\left(\mathbf{v}_{1}, \mathbf{v}_{2}\right)$ and $\left(\mathrm{id}_{\alpha}, \mathrm{id}_{\mu}\right)$ to obtain $\overline{\mathrm{id}}=\left(\mathrm{id}_{\alpha}, \mathrm{id}_{\mu}\right)$ and vectors $\mathbf{v}_{1}^{\prime}=\left(\hat{\mathbf{v}}_{1}, 1,0\right), \mathbf{v}_{2}^{\prime}=\left(\hat{\mathbf{v}}_{2}, 0,1\right)$. 
5. Run Combine $\left(\left(\mathbf{v}_{1}, \mathrm{id}_{\alpha}, \tau_{1}, 1\right),\left(\mathbf{v}_{2}, \mathrm{id}_{\mu}, \tau_{2}, 1\right)\right)$ to produce a signature $\tau_{4}$ on the vector $\mathbf{v}_{4}=\mathbf{v}_{1}^{\prime}+\mathbf{v}_{2}^{\prime}=\left(\hat{\mathbf{v}}_{1}+\hat{\mathbf{v}}_{2}, 1,1\right) \in \mathbb{F}_{p}^{n+2}$. Let $P_{4}=\left(\mathbf{v}_{4}, \overline{\mathrm{id}}, \tau_{4}\right)$.

6. Send $P_{3}$ and $P_{4}$ to Bob.

Receiver Bob. Bob receives $P_{3}$ and $P_{4}$, each of which pass the verification test (by the correctness of Sign and Combine). Bob then tries to decode the received data to recover Alice's file.

The identifier $\overline{\mathrm{id}}=\left(\mathrm{id}_{\alpha}, \mathrm{id}_{\mu}\right)$ indicates that $\mathbf{v}^{*}=\mathbf{v}_{4}-\left(\hat{\mathbf{v}}_{3}, 0,1\right)$ is a primitive vector sent by Alice, since the augmentation component of $\mathbf{v}^{*}$ is $(1,0)$. However, the data part of $\mathbf{v}^{*}$ is $\hat{\mathbf{v}}_{1}+\hat{\mathbf{v}}_{2}-\hat{\mathbf{v}}_{3}$, which cannot be in the subspace spanned by $\hat{\mathbf{v}}_{1}$ since $\left\{\hat{\mathbf{v}}_{1}, \hat{\mathbf{v}}_{2}, \hat{\mathbf{v}}_{3}\right\}$ are linearly independent. Thus $\mathbf{v}^{*}$ is an invalid vector accepted by Bob.

In the above attack, Mallet was able to frame Alice by secretly reusing $\mathrm{id}_{\mu}$ for two different vector spaces. Note that this attack is more insidious than simply inserting data with identifier $\mathrm{id}_{\alpha}$, which would have the same effect of corrupting Alice's data. We see from this attack that arbitrary file identifiers provide a malicious insider too much power. It is thus necessary to tie the identifiers cryptographically to the files they represent, in a way that is verifiable at every node in the network. In particular, the Sign algorithm should output both an identifier id and a signature $\sigma$. To verify the identifier we use an algorithm IdTest that takes as input a public key pk, a vector $\mathbf{y}$, and a list of identifiers id, and outputs "accept" if $\mathbf{y}$ is in the subspace $V$ identified by id. To avoid the above attack, the following tasks must be infeasible for Mallet:

1. Given a public key $\mathrm{pk}_{\alpha}$, find an identifier $\mathrm{id}_{\alpha}$ and a vector $\mathbf{y}$ such that $\operatorname{IdTest}\left(\mathrm{pk}_{\alpha}, \mathbf{y}, \overline{\mathrm{id}}_{\alpha}\right)$ outputs "accept." (This is a type of "collision-resistance" property.)

2. Given a vector space $V$, a public key $\mathrm{pk}_{\alpha}$, and $\left(\mathrm{id}_{\alpha}, \sigma\right):=\operatorname{Sign}\left(\mathrm{sk}_{\alpha}, V\right)$ (where $\mathrm{sk}_{\alpha}$ is the secret key corresponding to $\mathrm{pk}_{\alpha}$ ), find a $\mathbf{y} \notin V$ such that IdTest $\left(\mathrm{pk}_{\alpha}, \mathbf{y}, \mathrm{id}_{\alpha}\right)$ outputs "accept." (This property is unique to the network coding scenario.)

If Mallet can succeed at either task, then Bob is convinced that the vector $\mathbf{y}$ belongs to a file sent by Alice, when in fact it does not. (Indeed, in the first case Alice didn't even send a file!)

These two tasks are quite familiar: they are analogous to the two ways of breaking a single-source network coding signature scheme [4, Section 3.1]. This analysis leads to our key observation: the file identifier produced by Sign must itself be a vector space signature. It follows that all the security properties of the system are carried in the identifier id, so we can set the "signature" part $\sigma$ equal to id or eliminate $\sigma$ entirely. We formalize these ideas in the following section.

Remark 3. One can show that allowing the use of arbitrary file identifiers not only makes hop-by-hop containment impossible, but also forces the receiver to solve the clique problem for proper decoding. Specifically, there is a formal reduction from the clique problem to decoding in multi-source network coding; details are in the full paper [2]. 


\section{Network Coding Signatures}

We formally define the multi-source network coding signature scheme. Here the Sign algorithm generates an element $\sigma$ that is used both as a signature and a file identifier. The Verify algorithm implements the functionality of the IdTest algorithm in the previous section and allows every node to validate the identifier/signature of an incoming packet. Since signatures and identifiers play the same role, the Combine algorithm provides the same functionality as the Merge algorithm of Section 2, while also keeping track of the public keys involved. Note that in contrast to traditional signatures, the Verify algorithm does not take as input the original message (i.e., vector space).

Definition 4. A multi-source network coding signature scheme is a tuple of five PPT algorithms, Setup, KeyGen, Sign, Combine, Verify, with the following properties:

Setup $\left(1^{\lambda}, n, m\right)$ : On input the unary representation of a security parameter $1^{\lambda}$, a data space dimension $n$, and a subspace dimension $m$, outputs a description of system parameters params. This description includes the prime $p$ used to define the field over which vector spaces are defined, as well as $n$ and $m$.

KeyGen(params): Outputs a randomly generated user key pair (sk, pk).

Sign(params, sk, $V$ ): On input a secret key sk and a subspace $V \subset \mathbb{F}_{p}^{n+m}$, outputs a signature $\sigma$.

Combine(params, $\left.\left(\mathbf{v}_{1}, \boldsymbol{\sigma}_{1}, \overline{\mathrm{pk}}_{1}, a_{1}\right),\left(\mathbf{v}_{2}, \boldsymbol{\sigma}_{2}, \overline{\mathrm{pk}}_{2}, a_{2}\right)\right)$ : Takes as input two vectors $\mathbf{v}_{1} \in \mathbb{F}_{p}^{n+m f_{1}}$ and $\mathbf{v}_{2} \in \mathbb{F}_{p}^{n+m f_{2}}$, two lists of signatures $\boldsymbol{\sigma}_{1}, \boldsymbol{\sigma}_{2}$, two lists of public keys $\overline{\mathrm{pk}}_{1}, \overline{\mathrm{pk}}_{2}$, and two coefficients $a_{1}, a_{2} \in \mathbb{F}_{p}$. The algorithm outputs a list of signatures $\boldsymbol{\sigma}$ and a list of public keys $\overline{\mathrm{pk}}$.

Verify (params, $\overline{\mathrm{pk}}, \mathbf{v}, \boldsymbol{\sigma})$ : On input a list of public keys $\overline{\mathrm{pk}}$, a vector $\mathbf{v} \in \mathbb{F}_{p}^{n+m f}$, and a list of signatures $\boldsymbol{\sigma}$, outputs $\top$ (accept) or $\perp$ (reject).

Correctness. We require that for any set of system parameters determined by Setup $\left(1^{\lambda}, n, m\right)$, the following hold:

1. For primitive signatures: Consider a key pair (sk, pk) $\leftarrow$ KeyGen(params) and a vector space $V \subset \mathbb{F}_{p}^{n+m}$. Let $\sigma$ be the output of $\operatorname{Sign}($ params, sk, $V$ ). Let $\overline{\mathrm{pk}}=\{\mathrm{pk}\}$ and $\boldsymbol{\sigma}=\{\sigma\}$. Then for all $\mathbf{v} \in V$, we require that Verify (params, $\overline{\mathrm{pk}}, \mathbf{v}, \boldsymbol{\sigma})=\top$.

2. Recursively, for combined signatures: Consider two lists of public keys $\overline{\mathrm{pk}}_{1}, \overline{\mathrm{pk}}_{2}$, two vectors $\mathbf{v}_{1}, \mathbf{v}_{2}$, two lists of signatures $\boldsymbol{\sigma}_{1}, \boldsymbol{\sigma}_{2}$ such that

$$
\operatorname{Verify}\left(\text { params, } \overline{\mathrm{pk}}_{1}, \mathbf{v}_{1}, \boldsymbol{\sigma}_{1}\right)=\operatorname{Verify}\left(\text { params, } \overline{\mathrm{pk}}_{2}, \mathbf{v}_{2}, \boldsymbol{\sigma}_{2}\right)=\top \text {. }
$$

Let $\mathbf{v}_{1}^{\prime}, \mathbf{v}_{2}^{\prime}, \boldsymbol{\sigma}^{\prime}$ be the output of $\operatorname{Merge}\left(\mathbf{v}_{1}, \mathbf{v}_{2}, \boldsymbol{\sigma}_{1}, \boldsymbol{\sigma}_{2}\right)$. For any $a_{1}, a_{2} \in \mathbb{F}_{p}$, we require that if $\boldsymbol{\sigma}, \overline{\mathrm{pk}}$ is the output of the Combine algorithm on inputs $\left(\mathbf{v}_{1}, \boldsymbol{\sigma}_{1}, \overline{\mathrm{pk}}_{1}, a_{1}\right),\left(\mathbf{v}_{2}, \boldsymbol{\sigma}_{2}, \overline{\mathrm{pk}}_{2}, a_{2}\right)$, then: 
(a) $\sigma^{\prime}=\boldsymbol{\sigma}$

(b) For $j$ in $1, \ldots, f=|\boldsymbol{\sigma}|$, if the $j$ th element of $\boldsymbol{\sigma}$ is the $k$ th element of $\boldsymbol{\sigma}_{i}$ for $i \in\{1,2\}$, then the $j$ th element of $\overline{\mathrm{pk}}$ is the $k$ th element of $\overline{\mathrm{pk}}_{i}$.

(c) Verify (params, $\left.\overline{\mathrm{pk}}^{\prime}, a_{1} \mathbf{v}_{1}^{\prime}+a_{2} \mathbf{v}_{2}^{\prime}, \boldsymbol{\sigma}\right)=\top$.

In the second correctness condition, (a) tells us that identifiers and signature play the same role, while (b) requires that the list of public keys produced by Combine corresponds (in a natural way) to the list of identifiers produced by Merge.

\subsection{Security}

The security game captures the fact that if the system is secure, even an attacker who controls all sources but one and is given a chosen message oracle for the honest source cannot create an existential forgery on the honest source. The game between a challenger and an adversary $\mathcal{A}$ with respect to a signature scheme $\mathcal{S}$ proceeds as follows.

Init. The challenger runs $\operatorname{Setup}\left(1^{\lambda}, n, m\right)$ to obtain system parameters params and runs KeyGen(params) to obtain $\mathrm{sk}^{*}$ and $\mathrm{pk}^{*}$. It sends $\mathrm{pk}^{*}$ and params to $\mathcal{A}$. It keeps sk* to itself.

Signature queries. $\mathcal{A}$ adaptively requests signatures for vector spaces $V_{1}, \ldots, V_{\ell} \subset \mathbb{F}_{p}^{n+m}$. The challenger responds by computing Sign(params, $\left.\mathrm{sk}^{*}, V_{i}\right)$ for $i=1, \ldots, \ell$ and sends the resulting signatures to $\mathcal{A}$.

Forgery attempt. $\mathcal{A}$ eventually outputs a 4-tuple $\left(\overline{\mathrm{pk}}^{\dagger}, \mathbf{v}^{\dagger}, \boldsymbol{\sigma}^{\dagger}, W^{\dagger}\right)$, where $\overline{\mathrm{pk}}^{\dagger}$ is a list of $f$ (not necessarily distinct) public keys $\overline{\mathrm{pk}}^{\dagger}=\left(\mathrm{pk}_{1}, \ldots, \mathrm{pk}_{f}\right)$ that contains the challenge public key $\mathrm{pk}^{*}, \mathbf{v}^{\dagger}$ is a nonzero vector in $\mathbb{F}_{p}^{n+m f}, \boldsymbol{\sigma}^{\dagger}$ is list of $f$ signatures, and $W^{\dagger}=\operatorname{span}\left\{\mathbf{w}_{1}, \ldots, \mathbf{w}_{t}\right\} \subset \mathbb{F}_{p}^{n+t}$ for some $t$.

Adjudication. Let $\boldsymbol{\sigma}^{\dagger}=\left(\sigma_{1}, \ldots, \sigma_{f}\right)$ be the list of (distinct) identifiers output by $\mathcal{A}$, where, w.l.o.g. we assume the first $k$ components $\sigma_{1}, \ldots, \sigma_{k}$ are returned as the signatures for the chosen message queries $V_{1}, \ldots, V_{k}, k \leq \ell$. Let $\boldsymbol{\sigma}_{w}$ be the last $f-k$ elements of $\boldsymbol{\sigma}^{\dagger}$. Let $V^{*}$ be the vector space output by MergeSpaces $\left(V_{1}, \ldots, V_{k}, W^{\dagger}, \sigma_{1}, \ldots, \sigma_{k}, \boldsymbol{\sigma}_{w}\right)$.

The forger wins the game if Verify (params, $\left.\overline{\mathrm{pk}}^{\dagger}, \mathbf{v}^{\dagger}, \boldsymbol{\sigma}^{\dagger}\right)=\top$ and at least one of the following two conditions holds:

1. There exists $i$ in $1, \ldots, f$ such that the $i$ th component of $\mathrm{pk}^{\dagger}$ is equal to $\mathrm{pk}^{*}$, but $\sigma_{i}$ is not any of the signatures obtained in response to chosen message queries.

2. For $i=1, \ldots, t$, we have Verify $\left(\right.$ params, $\left.\overline{\mathrm{pk}}_{w}, \mathbf{w}_{i}, \boldsymbol{\sigma}_{w}\right)=\top$, but $\mathbf{v}^{\dagger} \notin V^{*}$.

Definition 5. The advantage $\mathrm{NC}-\operatorname{Adv}[\mathcal{A}, \mathcal{S}]$ of $\mathcal{A}$ is defined to be the probability that $\mathcal{A}$ wins the security game. A multi-source network coding scheme $\mathcal{S}$ is secure if for all probabilistic, polynomial-time adversaries $\mathcal{A}$ the advantage $\mathrm{NC}-\operatorname{Adv}[\mathcal{A}, \mathcal{S}]$ is negligible in the security parameter $\lambda$. 
In the security game, the attacker requests signatures for files $V_{1}, \ldots, V_{k}$ and creates his own file $W^{\dagger}$. Intuitively, $W^{\dagger}$ corresponds to the vector space (the set of files) whose data the adversary mixes with the honest user's data in order to frame the honest user. Winning condition (1) implies that the attacker can create a valid fake signature for one of the files that he requests signatures for, i.e., for a file signed with sk* . Winning condition (2) implies that the attacker can produce a fake file $W^{\dagger}$ whose basis vectors pass the verification test, and a vector $\mathbf{v}^{\dagger}$ that passes the verification test but lives outside the subspace $V^{*}$ that is the span of network coding combinations of the files he requested and created. A receiver that decodes the basis vectors of $W^{\dagger}$ together with the vector $\mathbf{v}^{\dagger}$ will be fooled into accepting a vector from the user with public key $\mathrm{pk}^{*}$ that this user never sent.

Implied properties. The security model implies that even given the secret key sk, no PPT adversary can construct distinct vector spaces $V_{1}, V_{2} \in \mathbb{F}_{p}^{n+m}$ such that $\operatorname{Sign}\left(\right.$ params, sk, $\left.V_{1}\right)=\operatorname{Sign}\left(\right.$ params, sk, $\left.V_{2}\right)$. Note, however, that this is no ordinary collision resistance property. During signature verification the vector space $V$ is not available and therefore the Verify algorithm must validate the signature given only $\mathbf{y} \in V$.

This collision resistance property is crucial during decoding. The decoder collects all incoming packets with a specific identifier into a full rank matrix and runs the decoding procedure. Collision resistance ensures that all packets with the same signature belong to the same vector space.

To see that this collision resistance property follows from our definition, it is not difficult to give a generic attack that works on any scheme for which this property is not satisfied. The attack, in fact, is essentially the same as the attack presented in Section 3.1 .

The vector space $W^{\dagger}$. Recall that the forgery attempt by the adversary consists of the 4-tuple $\left(\overline{\mathrm{pk}}^{\dagger}, \mathbf{v}^{\dagger}, \boldsymbol{\sigma}^{\dagger}, W^{\dagger}\right)$ where $\overline{\mathrm{pk}}^{\dagger}$ is a vector of public keys containing the challenge public key $\mathrm{pk}^{*}$. The other public keys in the vector $\overline{\mathrm{pk}}^{\dagger}$ are invented by the adversary and it is therefore possible that the adversary knows the corresponding private keys.

The vector $\mathbf{v}^{\dagger}$ and the signature $\boldsymbol{\sigma}^{\dagger}$ are the adversary's existential forgery. Suppose that $\left(\mathbf{v}^{\dagger}, \boldsymbol{\sigma}^{\dagger}\right)$ verify as a valid vector-signature pair with respect to $\overline{\mathrm{pk}}^{\dagger}$. We require the adversary to output the vector space $W^{\dagger}$ to prove that he is capable of exploiting $\mathbf{v}^{\dagger}$ to fool a recipient to incorrectly accept a vector from the single honest sender. To fool the recipient, the attacker can generate valid vectorsignature pairs for all basis vectors of $W^{\dagger}$ using the secret keys at his disposal. Since all these vectors have valid signatures, a recipient might try to decode the basis of $W^{\dagger}$ along with the vector $\mathbf{v}^{\dagger}$. If $\mathbf{v}^{\dagger} \notin V^{*}$, after decoding this set of vectors (i.e. after subtracting from $\mathbf{v}^{\dagger}$ the projection of $\mathbf{v}^{\dagger}$ onto $W^{\dagger}$ ), the recipient obtains a vector $\mathbf{u}$ that he believes came from the honest sender, but which the honest sender never sent since $\mathbf{u}$ is not in MergeSpaces $\left(V_{1}, \ldots, V_{k}, \sigma_{1}, \ldots, \sigma_{k}\right)$.

Hence, if the attacker is capable of producing a forgery for which condition (2) of adjudication holds, then an adversary can fool a recipient by sending it 
a sequence of properly signed vectors. We would like to require that for a secure signature scheme it should be impossible to produce a valid forgery where $\mathbf{v}^{\dagger} \notin V^{*}$. Unfortunately, this strong requirement appears to be unsatisfiable. We therefore weaken it to require that $\mathbf{v}^{\dagger} \notin V^{*}$ only when there is a possibility that the vectors in $W^{\dagger}$ will be jointly decoded with $\mathbf{v}^{\dagger}$, namely when Verify (params, $\left.\overline{\mathrm{pk}}_{w}, \mathbf{w}_{i}, \boldsymbol{\sigma}_{w}\right)=\top$ for all basis vectors $\mathbf{w}_{i}$ of $W^{\dagger}$. This is an acceptable weakening of the security requirement since the decoder will never group together vectors with different identifiers. In Section 5 we show that the resulting definition is satisfiable.

We note that requiring the adversary to output $W^{\dagger}$ is analogous to the security model of aggregate signatures [5] where the attacker outputs an aggregate signature from $s$ public keys, where $s-1$ of them are invented by the attacker. Moreover, the attacker must output the list of $s-1$ messages that went into the aggregate forgery, for each of the public keys the attacker invented. Our vector space $W^{\dagger}$ plays the same role as the $s-1$ messages in the aggregate forgery.

\section{Construction of a Multi-source Signature Scheme}

In this section, we construct an explicit multi-source network coding signature scheme satisfying Definition 4. In order to give a generic construction, we first define an auxiliary primitive called vector hash. This primitive captures the properties of the homomorphic hashes used by Krohn et al. 17] that are necessary for secure signatures.

\subsection{Vector Hashes}

A vector hash consists of three algorithms, Setup, Hash, Test, with the following properties:

HashSetup $\left(1^{\lambda}, n\right)$ : Input: unary representation of a security parameter $\lambda$ and dimension of the data space $n$. Output: public parameters pp.

Hash(pp, v): Input: public parameters pp and a vector $\mathbf{v} \in \mathbb{F}_{p}^{n}$. Output: hash $h$ of the vector $\mathbf{v}$. We require that this algorithm be deterministic.

Test $(\mathrm{pp}, \mathbf{y}, \bar{\beta}, \mathbf{h})$ : Input: Public parameters $\mathrm{pp}$, a vector $\mathbf{y} \in \mathbb{F}_{p}^{n}$, a vector of coefficients $\bar{\beta} \in \mathbb{F}_{p}^{m}$ and a vector of $m$ hash values $\mathbf{h}$. Output: $\top$ (true) or $\perp$ (false).

Let $\mathbf{h}$ be a set of hashes of a basis $\mathbf{v}_{1}, \ldots, \mathbf{v}_{m}$ of a vector space $V$. Intuitively, we want the Test algorithm to tell us whether $\mathbf{y}$ was constructed correctly from the basis, i.e., whether $\mathbf{y}=\sum \beta_{i} \mathbf{v}_{i}$. This means that Test should output $\top$ whenever $\mathbf{y}$ is constructed correctly, and it should be difficult for an adversary to find a vector $\mathbf{y} \notin V$ and a $\bar{\beta}$ such that Test outputs $T$. We now formalize these correctness and security conditions. 
Correctness. For correctness, we require the following for all public parameters $\mathrm{pp} \leftarrow \operatorname{HashSetup}\left(1^{\lambda}\right):$

1. For all $\mathbf{v} \in \mathbb{F}_{p}^{n}$, if $h \leftarrow \operatorname{Hash}(\mathrm{pp}, \mathbf{v})$ then we have Test(pp, $\left.\mathbf{v}, 1, h\right)=\top$.

2. Let $\mathbf{v} \in \mathbb{F}_{p}^{n}$, let $\bar{\beta} \in \mathbb{F}_{p}^{\ell}$ for some $\ell$, and let $\mathbf{h}$ be a list of hashes of length $\ell$. Fix $i \in\{0, \ldots, \ell\}$, let $\bar{\beta}^{\prime} \in \mathbb{F}_{p}^{\ell+1}$ be the vector $\bar{\beta}$ with a zero inserted between the $i$ th and $(i+1)$ th place, and let $\mathbf{h}^{\prime}$ be the vector $\mathbf{h}$ with any hash value inserted between the $i$ th and $(i+1)$ th place. We require that if $\operatorname{Test}(\mathrm{pp}, \mathbf{v}, \bar{\beta}, \mathbf{h})=\top$, then Test $\left(\mathrm{pp}, \mathbf{v}, \bar{\beta}^{\prime}, \mathbf{h}^{\prime}\right)=\top$.

3. Let $\mathbf{v}_{1}, \mathbf{v}_{2} \in \mathbb{F}_{p}^{n}$, let $\bar{\beta}_{1}, \bar{\beta}_{2} \in \mathbb{F}_{p}^{\ell}$ for some $\ell$, let $\mathbf{h}$ be a list of hashes of length $\ell$. Let $a, b \in \mathbb{F}_{p}$, let $\mathbf{y}=a \mathbf{v}_{1}+b \mathbf{v}_{2}$, and $\bar{\beta}=a \bar{\beta}_{1}+b \bar{\beta}_{2}$. We require that if $\operatorname{Test}\left(\mathrm{pp}, \mathbf{v}_{i}, \bar{\beta}_{i}, \mathbf{h}\right)=\top$ for $i=1,2$ then $\operatorname{Test}(\mathrm{pp}, \mathbf{y}, \bar{\beta}, \mathbf{h})=\top$.

Security. Let $\mathcal{V H}=($ HashSetup, Hash, Test) be a vector hash. Let $\mathcal{A}$ be a PPT algorithm that takes as input public parameters pp $\leftarrow \operatorname{HashSetup}\left(1^{\lambda}, n\right)$ and outputs a vector $\mathbf{v}^{*} \in \mathbb{F}_{p}^{n}$, an $m$-dimensional vector space $V \subset \mathbb{F}_{p}^{n}$ (for some $m$ ) represented as basis vectors $\mathbf{v}_{1}, \ldots, \mathbf{v}_{m}$, an $m$-tuple of coefficients $\bar{\beta}$, and a vector of hashes $\mathbf{h}=\left(h_{1}, \ldots, h_{m}\right)$.

Definition 6. With notation as above, we say that $\mathcal{A}$ breaks the vector hash scheme $\mathcal{V H}$ if $\mathbf{v}^{*} \notin V$, Test $\left(\mathrm{pp}, \mathbf{v}^{*}, \bar{\beta}, \mathbf{h}\right)=\top$, and Test $\left(\mathrm{pp}, \hat{\mathbf{v}}_{i}, \mathbf{e}_{i}, h_{i}\right)=\top$ for $i=1, \ldots, m$. (Recall that $\hat{\mathbf{v}}_{i}$ is the data component of $\mathbf{v}_{i}$.) We define the $a d$ vantage Hash- $\operatorname{Adv}[\mathcal{A}, \mathcal{V H}]$ of $\mathcal{A}$ to be the probability that $\mathcal{A}$ breaks $\mathcal{V} \mathcal{H}$. We say that a vector hash $\mathcal{V H}$ is secure if for all PPT algorithms $\mathcal{A}$ the advantage Hash- $\operatorname{Adv}[\mathcal{A}, \mathcal{V H}]$ is negligible in the security parameter $\lambda$.

In the full paper 2] we give an example vector hash using a finite cyclic group $\mathbb{G}$ of order $p$. This vector hash is secure if the discrete logarithm problem is infeasible in $\mathbb{G}$.

\subsection{The Construction}

For this construction, we use as a black box a vector hash as defined in Section 5.1.

Signature Scheme $\mathcal{N} \mathcal{S}$. Let $\mathcal{V H}=\left(\right.$ HashSetup $_{h}$, Hash $_{h}$, Test $\left._{h}\right)$ be a vector hash and let $\mathcal{S}=\left(\right.$ Setup $_{s}$, KeyGen $_{\mathrm{s}}, \operatorname{Sign}_{\mathrm{s}}$, Verify $\left._{\mathrm{s}}\right)$ be a signature scheme for signing messages in $\{0,1\}^{*}$. Our network coding signature scheme is as follows:

Setup $\left(1^{\lambda}, n, m\right)$ : Run HashSetup ${ }_{h}\left(1^{\lambda}, n\right)$ to obtain hash parameters and Setup $_{s}\left(1^{\lambda}\right)$ to obtain signature parameters. Let params contain $m, n$, and the outputs of these algorithms.

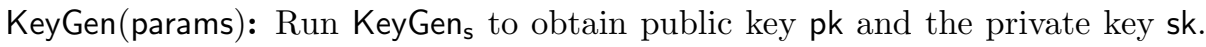
Output (pk, sk). 
Sign (params, sk, $\left.\left\{\mathbf{v}_{1}, \ldots, \mathbf{v}_{m}\right\}\right)$ : For $i=1, \ldots, m$, set $h_{i}:=\operatorname{Hash}_{h}\left(\right.$ params, $\left.\hat{\mathbf{v}}_{i}\right)$. Set $\mathbf{h}=\left(h_{1}, \ldots, h_{m}\right), \eta:=\operatorname{Sign}_{s}(\mathbf{s k}, \mathbf{h})$, and $\sigma:=(\mathbf{h}, \eta)$. Output $\sigma$.

Combine (params, $\left.\left(\mathbf{v}_{1}, \boldsymbol{\sigma}_{1}, \overline{\mathrm{pk}}_{1}, a_{1}\right),\left(\mathbf{v}_{2}, \boldsymbol{\sigma}_{2}, \overline{\mathrm{pk}}_{2}, a_{2}\right)\right)$ :

1. Let $\boldsymbol{\sigma}^{\prime}, \mathbf{v}_{1}^{\prime}, \mathbf{v}_{2}^{\prime}:=\operatorname{Merge}\left(\boldsymbol{\sigma}_{1}, \boldsymbol{\sigma}_{2}, \mathbf{v}_{1}, \mathbf{v}_{2}\right)$.

2. To create a list $\overline{\mathrm{pk}}^{\prime}$, do:

For $j$ in $1, \ldots, k=|\boldsymbol{\sigma}|$, if the $j$ th element of $\boldsymbol{\sigma}$ is the $k$ th element of $\boldsymbol{\sigma}_{i}$ for $i \in\{1,2\}$, then the $j$ th element of $\overline{\mathrm{pk}}^{\prime}$ is the $k$ th element of $\overline{\mathrm{pk}}_{i}$.

3. Output $\sigma^{\prime}$ and $\overline{\mathrm{pk}}^{\prime}$.

Verify (params, $\overline{\mathrm{pk}}, \mathbf{y}, \boldsymbol{\sigma})$ : Interpret $\boldsymbol{\sigma}$ as a list of $f$ signatures where each $\sigma_{i}=$ $\left(\mathbf{h}_{i}, \eta_{i}\right)$. Write $\mathbf{H}=\left(\mathbf{h}_{1}, \ldots, \mathbf{h}_{f}\right)$. Do the following:

1. For $i$ in $1, \ldots, f$, compute Verify ${ }_{s}\left(\mathrm{pk}_{i}, \mathbf{h}_{i}, \eta_{i}\right)$.

2. Compute $\operatorname{Test}_{h}$ (params, $\left.\hat{\mathbf{y}}, \bar{\beta}_{\mathbf{y}}, \mathbf{H}\right)$. (Recall $\bar{\beta}_{\mathbf{y}}$ is the augmentation component of $\mathbf{y}$.)

If all steps output $T$, output $T$; else output $\perp$.

The only difference between the Combine algorithm in our signature scheme and the Merge algorithm of Section 2.1 is that the Combine algorithm also keeps track of the public keys associated with the signatures.

Instead of sending a separate hash signature $\eta_{i}$ in each $\sigma_{i}$, we can aggregate these signatures together for space efficiency. In the full paper [2] we describe an instantiation of the system where a signature on $f$ files is of length $(f m+1) \log _{2} p$ bits. We also prove a lower bound showing that for large values of $f$ and $m$ this length is optimal.

Correctness. We verify the correctness conditions of Definition 4 .

1. For primitive signatures: Consider a key pair (sk, pk) $\leftarrow$ KeyGen(params) and a vector space $V \subset \mathbb{F}_{p}^{n+m}$ described by a properly augmented basis $\mathbf{v}_{1}, \ldots, \mathbf{v}_{m}$. Let $\sigma$ be the output of $\operatorname{Sign}\left(\right.$ params, sk, $V=\left\{\mathbf{v}_{1}, \ldots, \mathbf{v}_{m}\right\}$ ). Interpret the signature $\sigma$ as $\sigma=(\mathbf{h}, \eta)$.

For primitive signatures, there is only one file $f=1$. We examine each step of Verify in turn:

1. Since $\eta=\operatorname{Sign}_{s}(\mathrm{sk}, \mathbf{h})$, we have $\operatorname{Verify}_{s}(\mathrm{pk}, \mathbf{h}, \eta)=\top$ by correctness of $\mathcal{S}$.

2. Since $h_{i}=\operatorname{Hash}_{h}\left(\right.$ params, $\left.\hat{\mathbf{v}}_{i}\right)$, and $\beta_{\mathbf{v}_{i}}$ is the unit vector $\mathbf{e}_{i}$ since we are using a properly augmented basis, correctness conditions (1) and (3) of $\mathcal{V H}$ imply that $\operatorname{Test}_{h}\left(\right.$ params, $\left.\hat{\mathbf{v}}_{i}, \boldsymbol{\beta}_{\mathbf{v}_{i}}, \mathbf{h}\right)=\top$.

It follows that every basis vector $\mathbf{v}_{i}$ passes the signature verification test, i.e., Verify (params, pk, $\left.\mathbf{v}_{i}, \sigma\right)=\top$.

2. Recursively, for combined signatures: Consider two lists of public keys $\overline{\mathrm{pk}}_{1}, \overline{\mathrm{pk}}_{2}$, two augmented vectors $\mathbf{v}_{1}, \mathbf{v}_{2}$, two lists of signatures $\boldsymbol{\sigma}_{1}, \boldsymbol{\sigma}_{2}$ such that

$$
\operatorname{Verify}\left(\text { params, } \overline{\mathrm{pk}}_{1}, \mathbf{v}_{1}, \boldsymbol{\sigma}_{1}\right)=\operatorname{Verify}\left(\text { params, } \overline{\mathrm{pk}}_{2}, \mathbf{v}_{2}, \boldsymbol{\sigma}_{2}\right)=\top \text {. }
$$

Let $\mathbf{v}_{1}^{\prime}, \mathbf{v}_{2}^{\prime}, \boldsymbol{\sigma}^{\prime}$ be the output of $\operatorname{Merge}\left(\mathbf{v}_{1}, \mathbf{v}_{2}, \boldsymbol{\sigma}_{1}, \boldsymbol{\sigma}_{2}\right)$ and $f=|\boldsymbol{\sigma}|$. Let $\mathbf{H}_{i}$ be the list of all the hash elements in $\boldsymbol{\sigma}_{i}$ for $i=1,2$. Let $a_{1}, a_{2} \in \mathbb{F}_{p}$ be network 
combination coefficients, and let $\mathbf{y}=a_{1} \mathbf{v}_{1}^{\prime}+a_{2} \mathbf{v}_{2}^{\prime}$. Let $\boldsymbol{\sigma}, \overline{\mathrm{pk}}$ be the output of the Combine algorithm on inputs $\left(\mathbf{v}_{1}, \boldsymbol{\sigma}_{1}, \overline{\mathrm{pk}}_{1}, a_{1}\right),\left(\mathbf{v}_{2}, \boldsymbol{\sigma}_{2}, \overline{\mathrm{pk}}_{2}, a_{2}\right)$.

Conditions (a) and (b) are now immediate. For (c), we note that in our scheme, $\sigma_{j}^{\prime}=\left(\mathbf{h}_{j}, \eta_{j}\right)$ for $j=1, \ldots, f$. Let $\mathbf{H}=\left(\mathbf{h}_{1}, \ldots, \mathbf{h}_{f}\right)$. We examine each step of the Verify algorithm:

1. By the assumption (5.1) and the way we have set up the correspondence between indices of $\overline{\mathrm{pk}}$ and $\boldsymbol{\sigma}$, we have $\operatorname{Verify}_{s}\left(\mathrm{pk}_{j}, \mathbf{h}_{j}, \eta_{j}\right)=\top$ for $j$ in $1, \ldots, f$.

2. By assumption (5.1) we know that $\operatorname{Test}_{h}\left(\right.$ params, $\left.\hat{\mathbf{v}}_{i}, \bar{\beta}_{\mathbf{v}_{i}}, \mathbf{H}_{i}\right)=\top$ for $i=1,2$. By correctness property (2) of $\mathcal{V} \mathcal{H}$, for $i=1,2$ we have Test $_{h}\left(\right.$ params, $\left.\hat{\mathbf{v}}_{i}^{\prime}, \bar{\beta}_{\mathbf{v}_{i}^{\prime}}, \mathbf{H}\right)=\top$. Then, by correctness property (3) of $\mathcal{V H}$, we have $\operatorname{Test}_{h}\left(\right.$ params, $\left.\hat{\mathbf{y}}, \bar{\beta}_{\mathbf{y}}, \mathbf{H}\right)=\top$.

Thus, we have that Verify (params, $\left.\overline{\mathrm{pk}}^{\prime}, \mathbf{y}, \boldsymbol{\sigma}\right)=\top$.

We have the following security theorem; the proof is in the full paper 2].

Theorem 7. The network coding signature scheme $\mathcal{N S}$ is secure assuming that $\mathcal{V H}$ is a secure vector hash, and assuming $\mathcal{S}$ is a secure signature scheme.

\section{References}

1. Agrawal, S., Boneh, D.: Homomorphic MACs: MAC-based integrity for network coding. In: Abdalla, M., Pointcheval, D., Fouque, P.-A., Vergnaud, D. (eds.) ACNS 2009. LNCS, vol. 5536. Springer, Heidelberg (2009)

2. Agrawal, S., Boneh, D., Boyen, X., Freeman, D.M.: Preventing pollution attacks in multi-source network coding. Cryptology ePrint Archive (2010), Full version of this paper, available at http://eprint.iacr.org

3. Ahlswede, R., Cai, N., Li, S., Yeung, R.: Network information flow. IEEE Transactions on Information Theory 46(4), 1204-1216 (2000)

4. Boneh, D., Freeman, D., Katz, J., Waters, B.: Signing a linear subspace: Signature schemes for network coding. In: Jarecki, S., Tsudik, G. (eds.) Public Key Cryptography - PKC 2009. LNCS, vol. 5443, pp. 68-87. Springer, Heidelberg (2009)

5. Boneh, D., Gentry, C., Lynn, B., Shacham, H.: Aggregate and verifiably encrypted signatures from bilinear maps. In: Biham, E. (ed.) EUROCRYPT 2003. LNCS, vol. 2656, pp. 416-432. Springer, Heidelberg (2003)

6. Cai, N., Yeung, R.: Secure network coding. In: Proceedings of the 2002 IEEE International Symposium on Information Theory (2002)

7. Charles, D., Jain, K., Lauter, K.: Signatures for network coding. In: 40th Annual Conference on Information Sciences and Systems, CISS 2006 (2006)

8. Cohen, B.: Incentives build robustness in BitTorrent (2003), http://www.bittorrent.org/bittorrentecon.pdf

9. Feldman, J., Malkin, T., Stein, C., Servedio, R.: On the capacity of secure network coding. In: Proc. 42nd Annual Allerton Conference on Communication, Control, and Computing (2004)

10. Fragouli, C., Le Boudec, J.-Y., Widmer, J.: Network coding: an instant primer. SIGCOMM Comput. Commun. Rev. 36(1), 63-68 (2006)

11. Fragouli, C., Soljanin, E.: Network Coding Fundamentals. Now Publishers Inc., Hanover (2007) 
12. Han, K., Ho, T., Koetter, R., Médard, M., Zhao, F.: On network coding for security. In: Military Communications Conference, Milcom (2007)

13. Ho, T., Leong, B., Koetter, R., Médard, M., Effros, M., Karger, D.: Byzantine modification detection in multicast networks using randomized network coding. In: Proceedings of the 2004 IEEE International Symposium on Information Theory ISIT (June 2004)

14. Jaggi, S., Langberg, M., Katti, S., Ho, T., Katabi, D., Médard, M., Effros, M.: Resilient network coding in the presence of Byzantine adversaries. IEEE Trans. on Information Theory 54(6), 2596-2603 (2008)

15. Katti, S., Rahul, H., Hu, W., Katabi, D., Médard, M., Crowcroft, J.: XORs in the air: practical wireless network coding. IEEE/ACM Trans. Netw. 16(3), 497-510 (2008)

16. Koetter, R., Médard, M.: An algebraic approach to network coding. IEEE/ACM Transactions on Networking, 782-795 (2003)

17. Krohn, M., Freedman, M., Mazieres, D.: On the-fly verification of rateless erasure codes for efficient content distribution. In: Proc. of IEEE Symposium on Security and Privacy, pp. 226-240 (2004)

18. Li, Y., Yao, H., Chen, M., Jaggi, S., Rosen, A.: Ripple authentication for network coding. To appear in IEEE INFOCOM (2010), http://home.ie.cuhk.edu.hk/ mhchen/papers/ripple.infocom10.pdf

19. Zhao, F., Kalker, T., Médard, M., Han, K.: Signatures for content distribution with network coding. In: Proc. Intl. Symp. Info. Theory ISIT (2007) 